\title{
Codage et OFS
}

Pierre-François Cuénoud pour SwissDRG

René Raggenbass pour le domaine «Santé et Prévention» de la FMH
Dans son dernier bulletin du codage (Codinfo no 19 de décembre 2006, p. 8-9), l'Office fédéral de la statistique (OFS) a entériné - malgré nos protestations - sa décision unilatérale d'utiliser exclusivement le code F 17.1 du ICD-10 (Troubles mentaux et du comportement liés à l'utilisation du tabac, utilisation nocive pour la santé) pour décrire le tabagisme chronique. Le code F 17.2 qui concerne le syndrome de dépendance des mêmes troubles étant selon l'OFS à «bannir».

Le texte de l'OFS stipule clairement que la motivation de cette suppression du code F 17.2 est son utilisation abusive dans le système APDRG, de la part des hôpitaux: «Certains établissements en profitaient pour augmenter abusivement leur remboursement.» Il s'agit donc d'une mise en accusation très claire. Il est étonnant de voir que nos collègues allemands qui utilisent aussi l'ICD-10 pour définir leurs DRG n'ont pas eu à forcer la modification du système diagnostique sur ce point et qu'ils ne se plaignent pas d'abus de codage!

Même si l'OFS dit vouloir maintenant changer le libellé du code F 17.1 afin de quand même prendre en compte l'existence du syndrome de dépendance dans le tabagisme, la FMH proteste vivement contre cette modification, fondée sur une accusation grave et non démontrée, et contre ce procédé de décision unilatéral qui ne se fonde sur aucun critère clinique ou épidémiologique. De surcroît, le texte de l'OFS ne clarifie pas l'autre point que nous avions contesté, à savoir que seul les psychiatres psychothérapeutes étaient à même de poser un diagnostique de dépendance - ce que nous réfutons.

Ceci dit, la FMH attend de la part de l'OFS que cette modification forcée ne soit appliquée que transitoirement, et qu'elle soit réexaminée conjointement avec la FMH lors de l'introduction de la structure tarifaire SwissDRG. 